Nick BARON (dir.), Displaced Children in Russia and Eastern Europe, 19151953. Ideologies, Identities, Experiences, Leiden-Boston: Brill, Coll. Russian History and Culture, 2017, vol. 15, 310 p.

\title{
AleXANDRE SuMPF
}

\section{Université de Strasbourg}

Dans cet ouvrage collectif où il intervient à maints titres, Nick Baron ouvre un nouveau chapitre de sa participation à l'histoire des déplacements de population. Non content de signer une fertile introduction et une incisive conclusion, il livre l'un des articles les plus denses de cet ouvrage et a alimenté plusieurs auteurs en sources, réflexions et commentaires. Le recueil a pour objet le déplacement intentionnel et spécifique des enfants en tant que pratique étatique et expérience sociale, en se fondant sur des sources officielles et surtout sur des souvenirs inédits ou publiés. Les enfants subissent une épreuve violente multiforme, où alterne stigmatisation et protection, qui les transforme et les pousse à structurer une vision propre de l'origine, du transfert et de la réinstallation. L'État trouve chez les enfants autant une légitimation constitutive de son droit à intervenir directement dans la vie des citoyens qu'un laboratoire de transformation sociale. Ces expériences prennent en effet place au moment de l'essor d'une nouvelle conception médicale, psychologique et éducative de l'enfance, qui prend la forme d'un statut légal et de droits afférents. La prise de contrôle des vies d'enfants et d'adolescents se justifie alors en URSS par la criminalisation des attitudes de marginalité qui, parfois, découlent justement des déplacements : mendicité, délinquance, usage de drogues et rejet des normes sociales et de l'autorité.

Le recueil de dix articles possède une cohérence notable pour ce genre d'exercice, qui suggère l'intense travail collectif assuré par son coordinateur. Suivant la chronologie, les études de cas abordent d'abord les réfugiés lettons de la Grande Guerre (Aldis Purs), les exilés « blancs » de la guerre civile à Constantinople (Elizabeth White), les relégués « koulaks » en Sibérie (Nick Baron and Michael Kaznelson), les Républicains espagnols ayant trouvé refuge en URSS (Karl D. Qualls), les orphelins adoptés suite à la Seconde Guerre mondiale (Rachel Faircloth Green), les enfants soviétiques (Tara Zahra) ou juifs polonais (Gabriel N. Finder) déportés par les autorités allemandes, et enfin les Lituaniens du Goulag (Tomas Balkelis). Ce panorama des migrations forcées qui induisent tant de déplacements d'identité creuse un peu plus un sillon de l'histoire russo-soviétique déjà passablement labouré. Les sources convoquées permettent de révéler en partie la perception personnelle du déplacement/replacement de soi et du décalage des identités souvent situées dans le No man's land d'une transition sans fin.

Dans le chapitre d'Aldis Purs, les enfants lettons placés en orphelinat rédigent par exemple une autobiographie à la demande du directeur de l'institution, qui prend la liberté de les commenter à l'écrit. Mais l'État né de l'indépendance n'a pas intérêt à ce que leur voix porte un autre récit, moins héroïque et plus nostalgique, de la construction d'une identité nationale encore fragile. Michael Kaznelson s'est pour sa part rendu en 2003 à Narym pour y conduire dix entretiens avec des personnes âgées de plus de 70 ans, anciens relégués comme « koulaks » dans les années 1930. Leur récit et, plus largement, leur perception de leur propre histoire adoptent les catégories 
soviétiques et les codes du for intérieur à la soviétique mis en évidence par les travaux de Jochen Hellbeck. L'article, cosigné avec Nick Baron, révèle aussi une expérience différentielle entre les hommes qui ont pu négocier un nouveau départ en combattant dans les rangs de l'Armée rouge, et les femmes, cantonnées à l'identité imposée avant-guerre. Finder analyse, quant à lui, le dernier film en yiddish, Undzere Kinder (Nos enfants, 1949). L'intrigue mêle acteurs comiques juifs professionnels jouant leur propre rôle et jeunes enfants survivants jouant un rôle qui, sous le vernis de la captation documentaire, ne correspond qu'à une partie de leur histoire. Il ressort de ces exemples que la voix des enfants, même donnée pour directe et authentique, ne peut être légitimée que par le regard des adultes. Autocensuré, guidé ou instrumentalisé, le discours individuel sur leur passé sert surtout de fondement à un avenir dans lequel chacun doit impérativement se mouler.

Le titre de l'ouvrage prête un peu à confusion puisque le recueil concerne surtout la Russie/URSS et ses espaces coloniaux (baltes), avec un seul article consacré à la Pologne. Si l'Ukraine apparaît comme un champ de recherche fécond, que dire des peuples non slaves et non chrétiens (Coréens, Kazakhs, Tchétchènes, etc.) ou des communautés opprimées (Vieux-Croyants) ? Il me semble que le détour par des populations plus marginales - isolées, nomades, sans terre - permettrait d'affiner le rapport au lieu d'origine, désigné ici comme home/land. D'autre part, l'enfance est bien une construction sociale, mais elle ne doit pas faire oublier l'appartenance aux groupes sociaux. En effet, au-delà des étiquettes - Letton, koulak, orphelin, rescapé qu'en est-il des connaissances et de la sensibilité à l'histoire des enfants, qui sont autant des acquis culturels familiaux que des notions imposées par les États et les institutions ? Comment l'expérience du déplacement modifie-t-elle le rapport aux parents et aux frères et sœurs, la nuptialité, la parentalité ? Induit-elle une soviétisation de la famille?

D'autre part, si cette histoire prend sa source dans les défaites de l'armée tsariste pendant la Première Guerre mondiale, elle ne s'arrête pas vraiment en 1953 puisque certains souvenirs ont été publiés ou collectés justement à la fin du stalinisme, voire après la chute de l'URSS. Le choix de cette borne totémique apparaît d'autant moins logique que l'architecte de l'ouvrage développe une intéressante critique de l'usage des témoignages en histoire. Il aurait aussi été intéressant pour certains articles (Aldis Purs, Elizabeth White, Rachel Faircloth Green) d'adopter un temps plus long d'analyse, qui aurait par exemple permis la comparaison entre les deux sorties de guerre et entre les épisodes de répression - d'autant que « l'enfance » n'est pas immuable, puisqu'il s'agit d'une construction sociale.

Enfin, on regrette que nos collègues ignorent, comme souvent, les travaux non anglo-saxons sur les domaines qu'ils abordent, tels des Robinsons croyant leurs îles désertes. Le groupe des bezprizorniki que Baron place au centre du rapport entre État et société a été étudié en détail par Dorena Caroli. Ni Purs, ni White ne font référence aux écrits de Catherine Gousseff sur les enfants en guerre et leurs récits, ou sur la place singulière des enfants au sein de la diaspora russe.

Quant à Tomas Balkelis, il puise dans les souvenirs publiés en Lituanie après l'indépendance, mais se prive des possibilités offertes par le musée virtuel et européen du Goulag (gulagmemories.eu). D’une part, les interviews filmées offrent une autre 
idée des émotions ressenties et revécues ; d'autre part, les récits frappent par le rapport des enfants à la nature environnante, qu'ils habitent de leurs jeux et de leurs souvenirs, de leurs rêves et de leurs cauchemars. Le lieu de leur replacement n'est pas que passé et avenir, nostalgie et impératif de transformation : il existe au présent et, c'est sans doute la force de cet âge, est aussi un véritable lieu de vie. 